\title{
Immune responses to stroke: mechanisms, modulation, and therapeutic potential
}

\author{
Costantino ladecola, ${ }^{1}$ Marion S. Buckwalter, ${ }^{2}$ and Josef Anrather ${ }^{1}$ \\ ${ }^{1}$ Feil Family Brain and Mind Research Institute, Weill Cornell Medicine, New York, New York, USA. ²Department of Neurology and Neurological Sciences, Stanford University Medical Center, \\ Stanford, California, USA
}

\begin{abstract}
Stroke is the second leading cause of death worldwide and a leading cause of disability. Most strokes are caused by occlusion of a major cerebral artery, and substantial advances have been made in elucidating how ischemia damages the brain. In particular, increasing evidence points to a double-edged role of the immune system in stroke pathophysiology. In the acute phase, innate immune cells invade brain and meninges and contribute to ischemic damage, but may also be protective. At the same time, danger signals released into the circulation by damaged brain cells lead to activation of systemic immunity, followed by profound immunodepression that promotes life-threatening infections. In the chronic phase, antigen presentation initiates an adaptive immune response targeted to the brain, which may underlie neuropsychiatric sequelae, a considerable cause of poststroke morbidity. Here, we briefly review these pathogenic processes and assess the potential therapeutic value of targeting immunity in human stroke.
\end{abstract}

\section{Introduction}

Stroke remains one of the most prevalent and devastating diseases affecting the world population. Stroke is the second leading cause of death worldwide and the leading cause of long-term disability (1). Despite successes in the prevention and treatment of cardiovascular diseases (2), the lifetime risk of stroke increased from $22.8 \%$ in 1990 to $24.9 \%$ in 2016 (3). Another disturbing trend has been an increase in stroke incidence in the young (4), attributed to a surge in conventional stroke risk factors, such as hypertension, obesity, hyperlipidemia, smoking, and substance abuse (5).

Owing to aging of the population and reduced mortality due to improvements in acute stroke care, the prevalence of stroke is expected to increase from approximately $3 \%$ of the US population in 2012 to approximately $4 \%$ by 2030 , with an estimated annual cost approaching $\$ 200$ billion (6). Stroke survivors are at high risk for recurrent strokes and disabling long-term sequelae. Of these, one of the most troubling is cognitive impairment. Stroke survivors have double the risk of developing dementia (7), and ischemic lesions promote the expression of cognitive deficits in Alzheimer's disease, the major neurodegenerative dementia in the elderly (8). Since stroke can be prevented by control of vascular risk factors, there have been calls to reduce the impact of dementia by preventing stroke (9).

Approximately $70 \%$ of strokes are caused by occlusion of a major cerebral artery, usually the middle cerebral artery (3). Major causes of large arterial occlusion include thrombosis and embolism, most often caused by atrial fibrillation (10). Occlusion of small arteries and arterioles (small vessel disease) leads to small ischemic lesions in the basal ganglia and subcortical white matter

Conflict of interest: $\mathrm{Cl}$ serves on the Scientific Advisory Board of Broadview Ventures. Copyright: ( 2020, American Society for Clinical Investigation.

Reference information: J Clin Invest. 2020;130(6):2777-2788.

https://doi.org/10.1172/JCl135530.
(11). The other major type of stroke is cerebral hemorrhage, occurring in the brain substance (intraparenchymal hemorrhage) (12) or the subarachnoid space (subarachnoid hemorrhage) (13). While less frequent than ischemic stroke, hemorrhagic stroke is responsible for much of stroke's global burden, especially in low- and middle-income countries, where mortality rates approach 80\% (14).

This Review deals with ischemic stroke, specifically the role of immune factors. The brain harbors a wide variety of immune cells, which are essential for brain development and function (15, 16) and contribute to several neurological diseases, including stroke (17-19). Previously unrecognized interactions between the brain and systemic immunity have been identified in the acute and chronic phases of ischemic injury. Here, we provide a focused account of cerebral and systemic immunity's impact on the development of ischemic injury, its short- and long-term consequences, and the therapeutic approaches afforded by targeting the immune system. Finally, we will highlight outstanding questions to define a path forward for future studies.

\section{Overview of the ischemic cascade}

Our understanding of the mechanisms of cerebral ischemia has evolved considerably over time (Table 1). In the 1950s and 1960 s the prevailing notion was that stroke damage was rapid and irretrievable, leading to a therapeutic nihilism that lingers to this day. Attempts to surgically reopen occluded vessels immediately after stroke led to disastrous cerebral hemorrhages (e.g., ref. 20). In the 1970s and 1980s, it was observed that the reduction in cerebral blood flow (CBF) in the ischemic territory is not uniform. At the center of the ischemic territory (ischemic core), flow reduction ( $>80 \%$ of preischemic CBF) is so severe that it causes rapid cell death. However, at the periphery (ischemic penumbra), CBF is sufficient to keep neurons alive, even though they stop working to save energy $(21,22)$. Major pathogenic factors leading to cell death in the penumbra include glutamate excitotoxicity, calcium overload, 
Table 1. Evolution of the concept of ischemic injury and acute stroke management

\begin{tabular}{|c|c|c|c|}
\hline Year & 1940 s and 1950s & 1970 s and 1980 s & 1990s-present \\
\hline \multirow[t]{6}{*}{ Mechanism } & Energy failure & Core: energy failure & Core: energy failure \\
\hline & & Penumbra: Excitotoxicity/ $\mathrm{Ca}^{2+}$ & Penumbra: Excitotoxicity/Ca ${ }^{2+}$ \\
\hline & & Free radicals & Free radicals \\
\hline & & & Inflammation \\
\hline & & & Apoptosis \\
\hline & & & Genes \\
\hline Therapeutic window & $<30$ minutes & $1-2$ hours & $12-24$ hours \\
\hline \multirow[t]{3}{*}{ Management } & Anticoagulation & Anticoagulation & Stroke units \\
\hline & Endarterectomy & Stroke units & Thrombolysis \\
\hline & & & Mechanical thrombectomy \\
\hline Evidence based? & No & No & Yes \\
\hline
\end{tabular}

In the 1940 s and 1950s, rapid energy failure was considered the predominant injury mechanism, with a short therapeutic window. With the introduction of the concept of ischemic penumbra in 1977, the mechanisms were expanded to include glutamate excitotoxicity, $\mathrm{Ca}^{2+}$ overload, and free radical damage, and the therapeutic window was assumed to be longer. In the 1990s, changes in gene expression were found to drive inflammation and apoptosis in the postischemic brain. Since these processes developed hours to days after ischemia, the therapeutic window was felt to be longer. In contrast to these mechanistic advances, the mainstay of clinical stroke management has remained reperfusion therapy, largely unsuccessfully until the introduction of tPA and, more recently, mechanical thrombectomy. Stroke units were introduced in the mid 1970s, but their positive impact on stroke outcome was not demonstrated until recently (198).

tors on innate immune cells, enabling recognition of a wide variety of molecular complexes that are perceived as foreign and potentially damaging (dangerassociated molecular patterns [DAMPs]). Innate immune cells include neutrophils, monocytes, macrophages, and DCs, as well as selected groups of lymphocytes, e.g., NK cells, $\gamma \delta$ T cells, and others (33). The ensuing response is directed at eliminating the potential threat through a massive and indiscriminate humoral and cellular inflammatory response. In contrast to innate immunity, adaptive immunity requires several days to develop and retains memory of antigen exposure (32). Adaptive immunity is based on highaffinity receptors, including $\mathrm{T}$ cell receptors and immunoglobulins.

Innate and adaptive immunity are closely interrelated (32). Innate immune cells, DCs in particular, initiate adaptive immune responses via antigen presentation to lymphocytes, the typical adaptive immune cell. In turn, lymphocytes under-

and oxidative stress, and counteracting these factors leads to neuroprotection in animals $(22,23)$. In the 1990 s, ischemic cell stress was discovered to trigger molecular programs causing immune cell activation, inflammation, and programmed cell death. These pathogenic events promote the growth of the ischemic core into penumbral areas, expanding the injury to the entire ischemic territory (23-25). Therefore, cerebral ischemic injury results from a chain of events (ischemic cascade) that is triggered by the initial ischemic insult and unfolds over several hours or even days (26).

Despite these mechanistic advances, the only effective therapeutic approach has been to salvage the penumbra through reperfusion with the clot-busting drug tissue plasminogen activator (tPA) (27). More recently, successful recanalization has been achieved with mechanical thrombectomy in selected patients with penumbral tissue (refs. 28, 29, and Table 1). While remarkably effective, these treatments can be administered to fewer than $10 \%$ of stroke patients because of exclusion criteria, hemorrhagic complications, and highly specialized resources required for thrombectomy (30). However, advances in imaging the penumbra may provide the opportunity to rescue salvageable tissue using noninterventional means. For example, the recent ESCAPE trial, using a peptide that interferes with postsynaptic density protein 95 (PSD95), provided hints that neuroprotection is feasible in human stroke (31). Targeting the immune system is also an attractive strategy because of the anticipated extended therapeutic window of efficacy, low hemorrhagic complications, and involvement in both acute and chronic phases of the damage.

\section{Innate and adaptive immunity}

Innate and adaptive immunity represent the two main branches of the vertebrate immune system (32). Innate immunity is rapidly activated and relies on low affinity and promiscuous recep- go clonal expansion in lymphoid organs and return to the circulation to engage the antigen throughout the body. The resulting humoral and cellular responses seek to neutralize the offending antigen with a remarkable degree of selectivity and specificity. As detailed below, cerebral ischemia engages both innate and adaptive immunity (Figures 1 and 2), which play a critical role in both the acute and chronic phases of the damage.

\section{Cerebral ischemia and innate immunity: the brain view}

Circulating innate immune cells are quickly engaged at the onset of arterial occlusion, ultimately resulting in invasion of the ischemic brain by blood-borne immune cells and activation of brain-resident cells, which can be either beneficial or detrimental (Figure 1).

\section{Intravascular inflammatory events}

The sudden interruption of blood flow causing stroke leads to endothelial alterations, local trapping of blood cells, platelet-leukocyte adhesion, and activation of the coagulation cascade. The endothelium responds to changes in shear rates by expressing selectins, which loosely bind circulating leukocytes. Leukocyte adhesion takes place in venules within minutes after reperfusion in focal ischemic models, although transmigration occurs only after the blood-brain barrier (BBB) is compromised and parenchymal DAMP and chemokine gradients are established (34). Adhering leukocyte and platelets may clog vessels and have been implicated in the "no reflow" phenomenon that prevents reperfusion after the occlusion is released (ref. 35; but see also ref. 36). Thrombin induces expression of adhesion molecules on endothelial cells through activation of protease-activated receptors (37), acts as a chemotaxin for leukocytes, disrupts endothelial barrier function, and activates both C3 and C5 components of the complement system (38). 
A

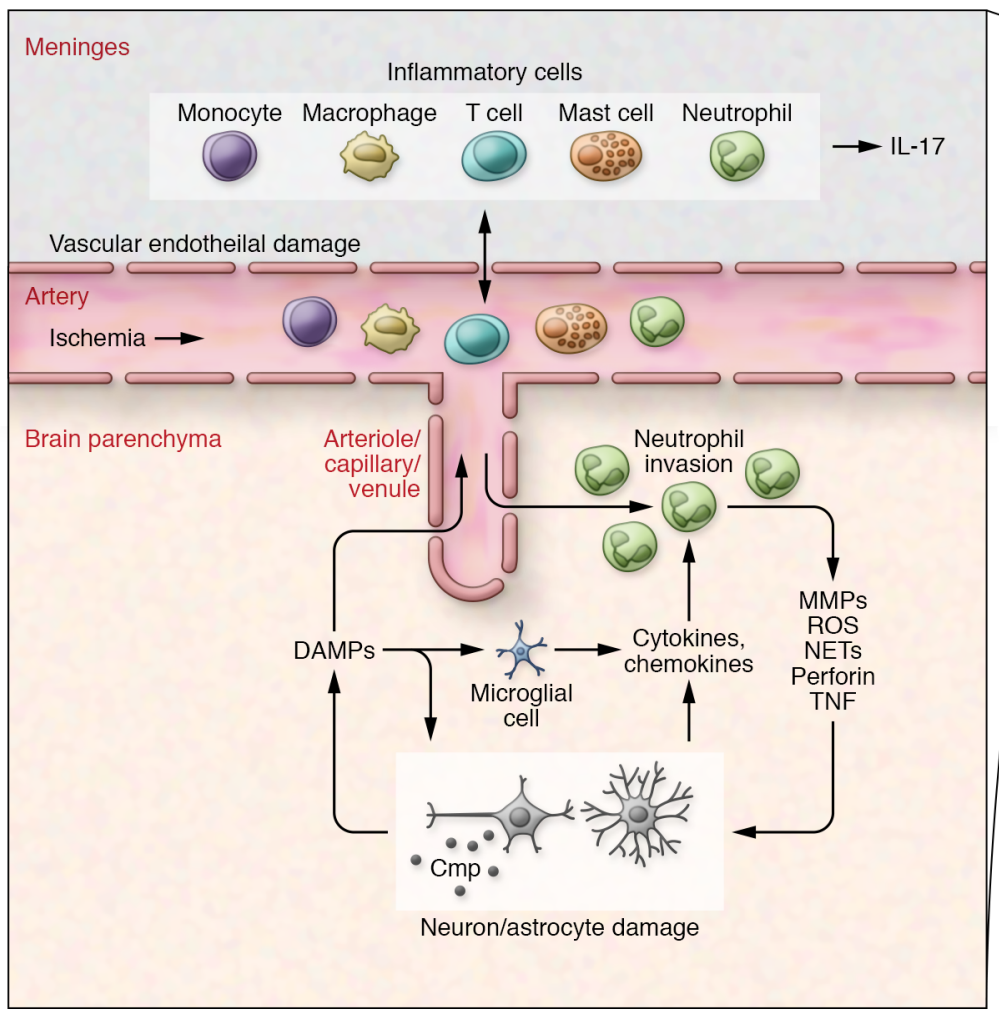

B

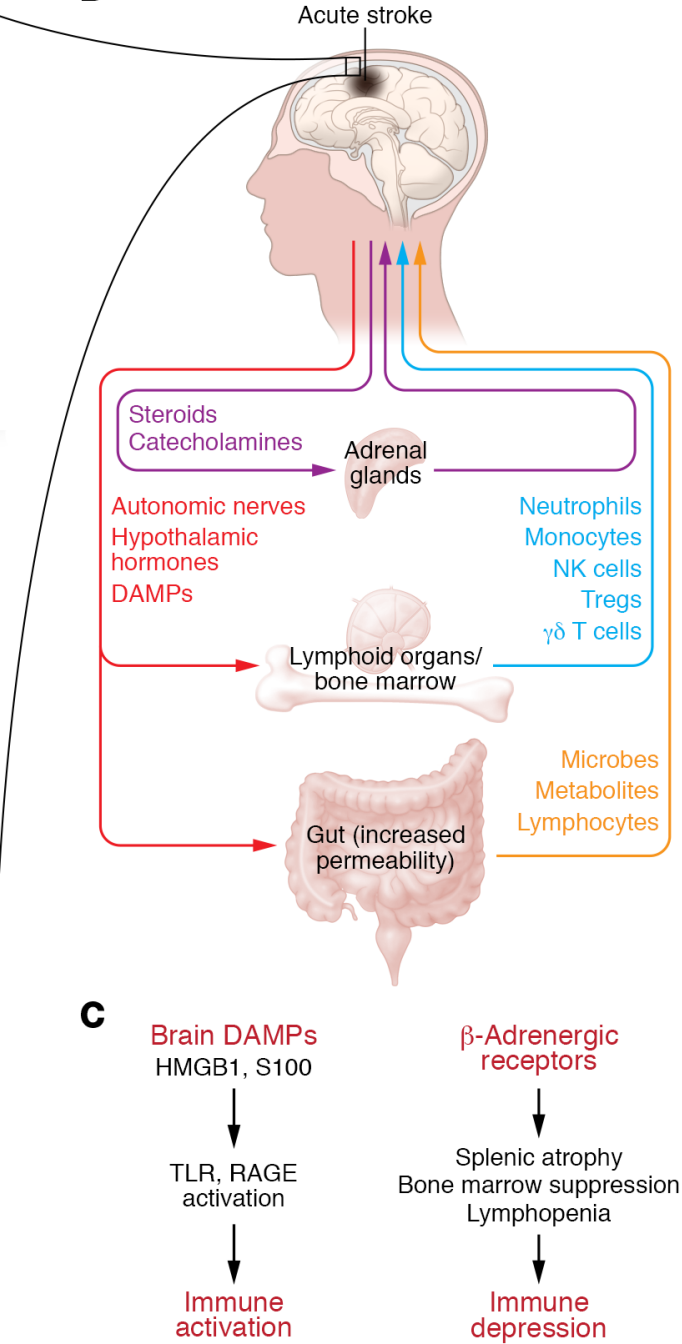

Figure 1. Cerebral and systemic immune changes in acute stroke: innate immunity. (A) After ischemia, circulating white cells stick to the cerebral endothelium and extravasate into the brain and meninges. Recent evidence implicates the skull bone marrow as a source of meningeal inflammatory cells (101). Cerebral ischemia also damages brain cells, which release DAMPs. DAMPs activate innate immune receptors on microglia and other cells, leading to the release of cytokines and chemokines, which, in turn, promote additional neutrophil entry. Neutrophils damage the brain by producing ROS, metalloproteases (MMPs), perforins, cytokines, and neutrophil extracellular traps (NETs). Activation of the complement cascade (Cmp) also damages brain cells. (B) Brain damage triggers a neurohumoral response (via the hypothalamic-hypophyseal axis and autonomic nervous system), which leads to activation of the adrenal glands and secretion of glucocorticoids and catecholamines. Brain-derived DAMPs leak into the circulation and activate systemic immunity, mobilizing innate immune cells form lymphoid organs and the gut. The increase in gut permeability may release bacteria and their metabolites into the circulation. (C) DAMPs activate systemic immunity through pattern recognition receptors, including TLRs and RACE, on immune cells. This activation phase is followed by immunodepression, attributable mainly to the systemic effects of $\beta$-adrenoreceptors, which increases the propensity to post-stroke infections.

\section{Complement}

Activation of the complement system, the humoral branch of innate immunity, is consistently associated with unfavorable stroke outcome (39) through opsonins (iC3b, C3dg, C3d) and anaphylatoxins (C3a, C5a). While intravascularly generated, active complement components might gain access to the brain parenchyma through a compromised BBB, complement synthesis also increases in microglia (40). Anaphylatoxins act on complement receptors on myeloid cells to promote free radical production, secretion of proinflammatory cytokines, degranulation, and phagocytosis. The C3a receptor is implicated in stroke, and genetic deletion or receptor antagonists reduce stroke and improve functional outcome (41). The lectin pathway initiates complement cascades and may also contribute to ischemic injury. Mice deficient in mannose-binding lectin, the major activator of the lectin cascade, were protected from ischemic injury (42), whereas deficiency in humans correlated with better stroke outcome $(43,44)$. Complement was also implicated in the chronic phase of damage. Injured neurons bind to naturally occurring IgMs that recognize DAMPs, such as modified annexin IV. The ensuing complement activation promotes the phagocytosis of stressed neurons by microglia/macrophages (45).

\section{Brain-resident immune cells}

After ischemia, microglial activation occurs before the appearance of neuronal cell death (46). Early responses are characterized by increased arborization and exploratory behavior, followed by dear- 
A

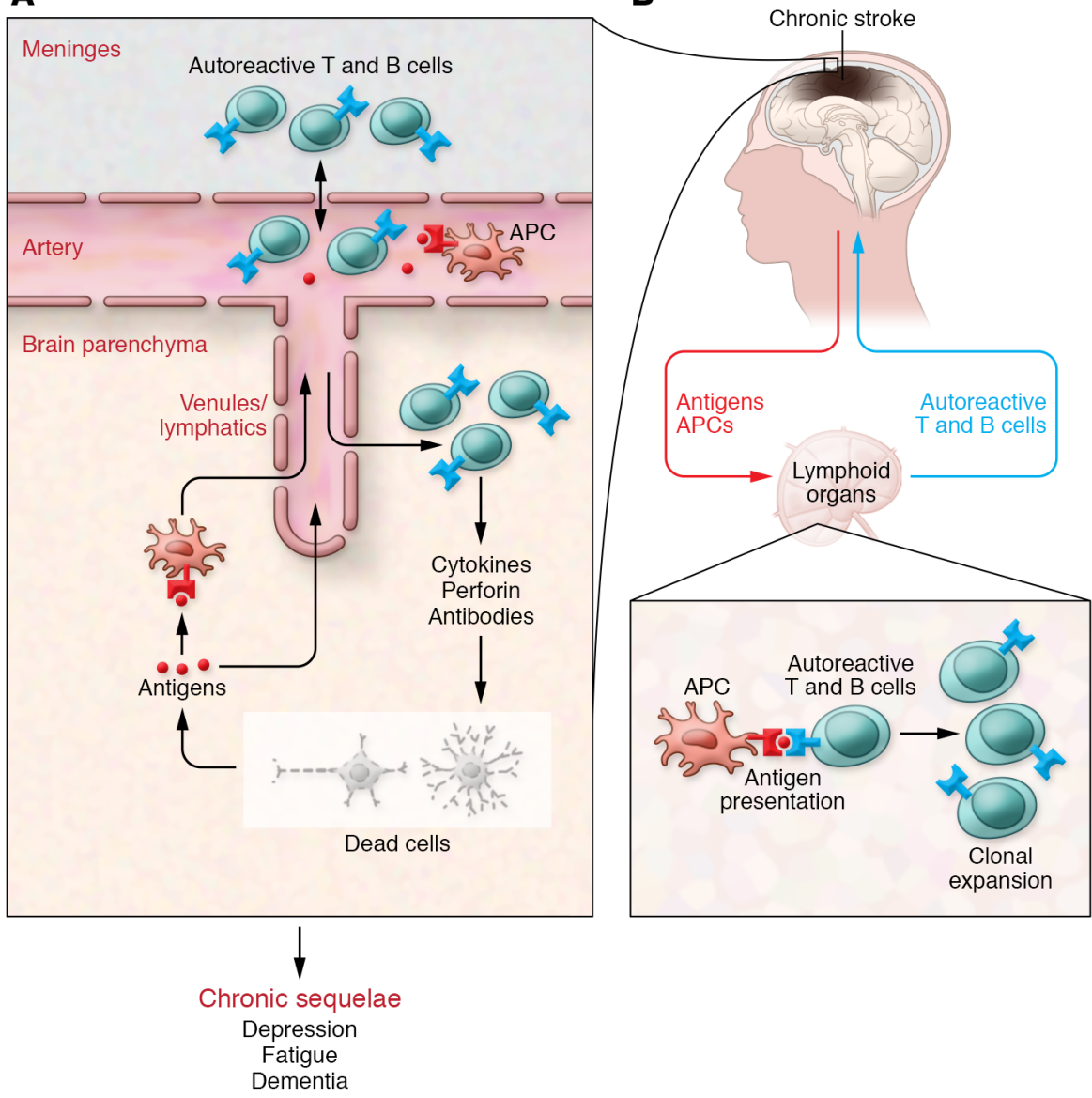

Figure 2. Cerebral and systemic immune changes in the chronic phase of stroke: adaptive immunity. (A) With tissue damage, dead cells release new antigens that may enter into contact with antigen presenting cells (APCs) in the brain. (B) Although not firmly established, these cells may enter the circulation and home into peripheral lymphoid organs. At the same time, antigens also reach the circulation and are detected by APCs in lymphoid organs. APCs, in turn, engage naive lymphocytes, which undergo differentiation ( $T$ or B cells) and clonal expansion, and reenter the circulation. These "autoreactive" lymphocytes, sensitized against brain antigens, home back into the brain and cause chronic inflammation and cytotoxicity, which may underlie the chronic sequelae of stroke. borization and ameboid transformation within hours after stroke onset (47). Microglia contribute to phagocytosis during the first days after cerebral ischemia, before the brain infiltration of bloodborne macrophages (48). While microglia are proinflammatory and exhibit cytotoxic activity in vitro, microglial depletion worsens stroke outcome $(49,50)$. Perhaps microglial cells limit poststroke inflammation by providing the neurotrophic factor IGF-1, phagocytizing dead cells and neutrophils, and suppressing astrocyte activation (49-52). Astrocytes participate in the immune response to ischemia by upregulating proinflammatory genes and promoting neutrophil recruitment through $\mathrm{C}-\mathrm{X}-\mathrm{C}$ class chemokines $(53,54)$.

The role of other brain-resident immune cells, including meningeal and perivascular macrophages, is less well understood. Brain macrophage depletion prior to stroke decreases BBB disruption in meningeal vessels and modestly ameliorates functional deficits without affecting infarct volume (55). The meninges also contain a sizable population of mast cells primarily located in the dura mater (ref. 56 and Figure 1). Mast cells contain granules with vasoactive agents and proteases and have been implicated in BBB disruption and neutrophil extravasation in cerebral ischemia, and deficiency (in $\mathrm{Kit}^{-/-}$mice) or pharmacologic inhibition of mast cells confers neuroprotection $(56,57)$. Neurons may also contribute to disruption of immune homeostasis after stroke. Neuronal death results in loss of the CSF1R ligand IL-34 $(58,59)$, which contributes to microglia depletion, whereas loss of the neuronal fractalkines CX3CL1 and CD200 increases microglial activation after stroke $(60,61)$. Although the CC3CL1/CX3CR1 pathway has been shown to contribute to ischemic injury in mice (62-64), whether this is due to changes in neuronal-microglial interactions remains to be established.

\section{Peripheral immune cells}

Neutrophils. Neutrophils are among the earliest immune cells recruited into the ischemic brain. Neutrophils contribute to ischemic damage by releasing proteases including elastase, metalloproteases (MMP9), cathepsin G, reactive oxygen and nitrogen species, and inflammatory IL-1 $\beta$, and by forming neutrophil extracellular traps consisting of extruded nuclear or mitochondrial DNA scaffolds decorated with proteases and cytotoxic histones (65-68). However, neutrophils that show signs of alternative activation, associated with the expression of Arg1 and YM1, might be beneficial in cerebral ischemia. For example, since cytotoxic neutrophils are generated through TLR4 signaling, it was proposed that neuroprotection observed after myeloid-selective TLR4 deletion is due to cytoprotective neutrophils $(69,70)$.

Myeloid cells. Monocyte-derived macrophage (MDM) influx into the ischemic brain requires chemokine signaling (CCL2/ CCR2), and blocking MDM entry exacerbates the outcome of cerebral ischemia (71-73). Once in the ischemic brain, MDMs exhibit remarkable plasticity, downregulating CCR2 and increasing CX3CR1 expression, but the consequence of this switch remains unclear (71-73). Early after ischemia, MDMs express markers of 
alternative activation, including Arg1, YM1 (Chil3), and CD206 (Mrc1) (74), consistent with a protective role. However, later on, they exhibit a proinflammatory phenotype that may contribute to chronic inflammation (74-76). Some MDMs are closely associated with blood vessels $(71,75)$, and $C c r 2^{-/-}$mice, in which postischemic MDM influx does not occur, showed reduced expression of TGF- $\beta 1$, thrombospondin, and collagen-4, leading to vascular instability, hemorrhagic transformation, and reduced angiogenesis $(72,77)$, suggesting a vasoprotective role. The spleen is a source of brain MDMs $(78,79)$ and may be important for regulating the immune response after stroke (80-82).

Dendritic cells. DCs are antigen-presenting cells with high migratory capacity, derived from dendritic (conventional cDC1 and cDC2 and plasmacytoid DC) or monocytic (moDC) lineages (83). DCs are found in the ischemic brain early after injury and persist for at least 7 days (84). Xcr1 ${ }^{-} \mathrm{CD} 172^{+} \mathrm{CDC} 2 \mathrm{~s}$ were identified as the major population contributing to ischemic brain injury in mice; this subtype secretes IL-23, inducing IL-17 expression in $\gamma \delta \mathrm{T}$ cells and neutrophil infiltration (85). CD209+ DCs have been observed in human poststroke brains up to 4 weeks after onset. DCs showed perivascular location and were in close proximity to T cells, indicating possible antigen presentation within the ischemic territory (86).

Lymphocytes and NK cells. T cells are detrimental early after ischemia, and lymphocyte-deficient mice are protected in models of focal ischemia $(87,88)$. The mechanism does not involve antigen-mediated $\mathrm{T}$ cell activation, and cytotoxic activity may be tied to innate $\mathrm{T}$ cell functions (88). Accordingly, natural IL-17secreting $\gamma \delta \mathrm{T}$ cells contribute to ischemic injury by inhibiting neutrophil infiltration $(53,85,89,90)$. NK cells also contribute to ischemic brain injury (91). CX3CR1 expression on NK cells is required for neutrophil recruitment, which depends on IFN- $\gamma$ and perforin expression, pointing at a direct neurotoxicity of NK cells (91). While effector lymphocytes may contribute to focal ischemic injury, Tregs appear in ischemic tissue after the acute phase and confer neuroprotection by downregulating postischemic inflammation via IL-10 (92).

\section{Entry points of peripheral immune cells}

Blood-brain barrier. The BBB's first opening, due to increased transcytosis, occurs within hours after cerebral ischemia (93). The second opening occurs 24 to 48 hours later and is characterized by proteolytic degradation of tight junctions and basement membranes, and loss of vascular cells, including endothelial cells, pericytes, and astrocytic end-feet (94). During this later phase, substantial infiltration of peripheral immune cells is observed. However, neutrophils are often found stuck around blood vessels (perivascular cuffs), which could be due to the barrier function of the glia limitans or to a lag in the implementation new transcriptional programs enabling parenchymal entry (65), which relies on the scavenger receptor CD36 and CSF3 in cerebral endothelial cells (95).

Meninges. Neutrophils are found on the abluminal site of leptomeningeal vessels within hours after experimental stroke (96). Tissue samples of human stroke victims reveal a strong association of neutrophils with leptomeningeal vessels (97). Whether meningeal neutrophils go on to infiltrate the ischemic territory remains to be established, but their entry is supported by the fact that accumulation in the meninges precedes their appearance in the brain parenchyma (97-99). Based on the observation that vascular channels connect the dura mater with the skull bone marrow, it has been proposed that leukocytes, in particular monocytes and neutrophils, may enter the brain through this alternative route $(100,101)$.

Choroid plexus. Owing to a fenestrated endothelium, the choroid plexus is the entry site for patrolling lymphocytes - mostly $\mathrm{CD}^{+}$central memory $\mathrm{T}$ cells - in the healthy brain (102). To promote leukocyte trafficking, the choroid plexus epithelium constitutively expresses ICAM-1 and VCAM-1, which, together with the mucosal vascular adhesion molecule MAdCAM-1, are upregulated in stroke (103). Choroid plexus epithelial cells also express the chemokine CCL20, which acts on CCR6 on the surface of IL-17-secreting lymphocytes to enable their entry into the cerebrospinal fluid (CSF) (104). Monocytes enter the CSF through the choroid plexus after ischemic stroke (105). CD73, an ecto-ATPase expressed on choroid plexus epithelial cells and lymphocytes, mediated monocyte/macrophage trafficking across the choroid plexus in a model of spinal cord injury (106). CD73 deletion exacerbated ischemic brain injury in mice, indicating that the choroid plexus could be an entry site for "neuroprotective" monocytes (107). In addition, the stroma of the choroid plexus contains brain-resident macrophages and DCs expressing MHC class II molecules, which may present antigens to T cells (108).

\section{Cerebral ischemia and innate immunity: the systemic view}

After brain damage, DAMPs and cytokines leak into the circulation and activate systemic immunity. Brain damage also activates neurohumoral pathways, which contribute to immune activation and inflammation and, later, immunodepression (Figure 1).

\section{Stroke and lymphoid organs: immune activation to immunodepression} Brain-generated DAMPs and cytokines produced during ischemic injury's early phase reach the systemic circulation through disrupted BBB or CSF drainage lymphatic pathways (109). In animals as in humans, circulating HMGB-1 increases with acute stroke, and blocking the HMGB-1/RAGE axis confers protection in animals $(110,111)$. Circulating DAMPs and cytokines induce an immune response in lymphoid organs, triggering inflammation. For example, signals from the postischemic brain reach cervical lymph nodes and promote systemic inflammation through VEGF3 receptors (112). In experimental stroke, this immune response is characterized by elevated serum cytokines (IL-6, IFN- $\gamma$, CXCL1) and increased production of inflammatory mediators in circulating and splenic immune cells (TNF, IL-6, IL-2, CCL2, and CXCL2) within hours after ischemia $(113,114)$. This response is transient and subsides within 24 hours after stroke. Comparable changes can be observed in stroke patients. TNF and IL- 6 are increased at stroke onset ( $<24$ hours) (115), and IL-6 serum levels correlate with stroke severity and unfavorable outcome (116). Clinical studies also report acute modifications in the transcriptome of circulating innate immune cells $(117,118)$, and experimental studies demonstrate differences in immune cell compartments in bone marrow, spleen, lymph nodes, and gut (119).

Postischemic immune activation is followed by immunodepression, which is linked to poststroke infections, a major cause of morbidity and mortality (120). Early studies on the immune 
status of stroke patients found prolonged peripheral lymphopenia and reduced $\mathrm{T}$ cell responsiveness $(121,122)$. Similarly, loss of circulating and splenic B, T, and NK cells was observed after experimental stroke (123). Mechanistically, this involves activation of the autonomic nervous system and the hypothalamic-adrenal axis, leading to the release of norepinephrine, acetylcholine, and glucocorticoids (refs. 123-125 and Figure 1). These mediators act synergistically to induce splenic atrophy, T cell apoptosis, and NK cell deficiency via norepinephrine and glucocorticoids. In the bone marrow, tyrosine hydroxylase and norepinephrine increase 1 day after stroke in mice (126). This triggers a response in mesenchymal stromal cells, through $\beta_{3}$-adrenergic receptors, suppressing cell retention factors such as IL-7, CXCL12, VCAM-1, and angiopoietin-1. Downregulation of these factors promotes myeloid lineage proliferation, suppresses lymphoid lineage progression, and results in lymphopenia (126). The sympathetic nervous system also plays a role in this response. In mice, $\beta$-adrenergic receptor inhibition lowers bacteremia and lung colonization and increases survival rates along with preservation of splenic and circulating lymphocytes (123). Vagus nerve stimulation or activation of $\alpha 7$-nicotinic acetylcholine receptor is protective in focal cerebral ischemia (127-130), but it also increases pulmonary infections, thus limiting its translational value (131).

\section{The gut-brain axis in stroke}

Conventional lymphocytes and $\gamma \delta$ T cells traffic from small intestine to brain and meninges after stroke, where they exacerbate ischemic injury $(89,132)$. Neurohumoral signals generated by the ischemic brain in turn affect immune homeostasis, intestinal barrier function, and microbiota. Importantly, up to $50 \%$ of stroke patients experience gastrointestinal complications, including altered motility, microbial dysbiosis, and intestinal bleeding, which are associated with increased mortality rates and impaired functional recovery (133). In mice, $\beta$-adrenergic signaling disrupted intestinal mucin production, increased gut permeability, and changed microbial composition after stroke $(132,134)$. Gut barrier disruption was associated with increased bacterial translocation and seeding of intestinal bacteria to blood, liver, and lungs (135). Interestingly, aged mice were more susceptible to bacterial translocation and failed to clear bacteria owing to a more severe poststroke immunosuppression (136). A recent study identified TREM1 upregulation in intestinal macrophages as a possible mediator of intestinal barrier disruption caused by $\beta$-adrenergic signaling (137).

\section{Adaptive immunity, chronic inflammation, and long-term stroke sequelae}

Increasing evidence indicates that after stroke, antigen presentation engages adaptive immunity. The next sections discuss adaptive responses affecting the postischemic brain and their role in the neuropsychiatric sequelae of stroke.

\section{Adaptive immunity and stroke}

Starting immediately after the stroke, novel brain antigens generated by cell stress and damage reach the circulation and stimulate $\mathrm{B}$ and $\mathrm{T}$ lymphocytes in spleen and lymph nodes. Concurrently, dead cells are also phagocytosed by myeloid cells in the brain, which upregulate the MHC class II to become antigen-presenting cells, then migrate to lymph nodes and spleen (138). This antigen presentation process is likely to occur both inside and outside the brain (Figure 2). Indeed, $\mathrm{T}$ cells migrate to the ischemic region in an antigen-independent fashion within days after stroke and are present in the lesion (90). However, a recent study reported that Tregs with a brain-specific signature accumulated during the chronic phase of stroke independently of antigen presentation (139). The Tregs expanded locally, and their function required IL-2, IL-33, and serotonin signaling via the HTR7 receptor. Tregs limited astrogliosis, suppressed the neurotoxic phenotype of astrocytes, and promoted functional recovery without affecting infarct volume. Similarly, although B lymphocytes are not present in large numbers in the brain early after stroke (140), regulatory B cells are protective (141) and may also increase beneficial Treg activity (142).

Antigen-dependent autoimmunity is also present after stroke (143). Autoreactive $\mathrm{CD} 4^{+}$and $\mathrm{CD} 8^{+} \mathrm{T}$ cells and B cells increase 4 days after stroke (144). In stroke survivors, peripheral blood lymphocytes tested in the second week after stroke demonstrate more activity against myelin than those in controls or even people with multiple sclerosis (145). This autoimmunity, similar to antigenindependent responses, can be harmful or beneficial dependent on polarization of $\mathrm{T}$ lymphocytes. For example, prior tolerization of Tregs specific to the brain antigen myelin oligodendrocyte glycoprotein (MOG) leads to IL-10-dependent neuroprotection after stroke (146). Conversely, a proinflammatory stimulus after stroke, such as LPS in rats or infection in humans, will lead to a detrimental Th1 response against brain antigens and worse outcomes (147, 148). Late B lymphocyte responses also occur after stroke, and CNS antibody production may increase over time, with about half of stroke survivors exhibiting intrathecal antibody synthesis after the first week (149-151). However, the duration of this response and its impact on outcome remain unclear.

\section{Poststroke dementia}

There are tantalizing links between adaptive immunity, chronic inflammation, atrophy, and dementia after stroke (Figure 2). In humans, inflammation persists in the blood months after stroke $(152,153)$, and can last much longer in the brain. In about half of stroke survivors, activated microglia and astrocytes, foam cells, and lymphocytes in the stroke are present decades after the event (140, 154). Interestingly, ischemia-induced inflammation is longer lasting in the brain than in the heart (155). Chronic inflammation is neurotoxic, and the glial scar that surrounds it is devoid of tight junctions in both humans and mice $(155,156)$. It is permeable to albumin and antibodies, perhaps causing peristroke neuronal injury (156).

In humans, the link between brain atrophy and chronic brain inflammation is not well established. Poststroke atrophy has been attributed to loss of network integrity that causes atrophy of connected regions (157). Brain atrophy can also occur from stroke risk factors in the absence of clinical stroke (158-162), and medial temporal atrophy, which may occur prior to stroke, is associated with cognitive decline after stroke (163). However, high C-reactive protein levels within 72 hours of stroke were linked to hippocampal atrophy and worse cognition one year later (164). In mice, atrophy also occurs late after stroke and is partially dependent on the proinflammatory cytokine osteopontin, although a role for lost connectivity among brain regions cannot be ruled out $(155,156)$. 


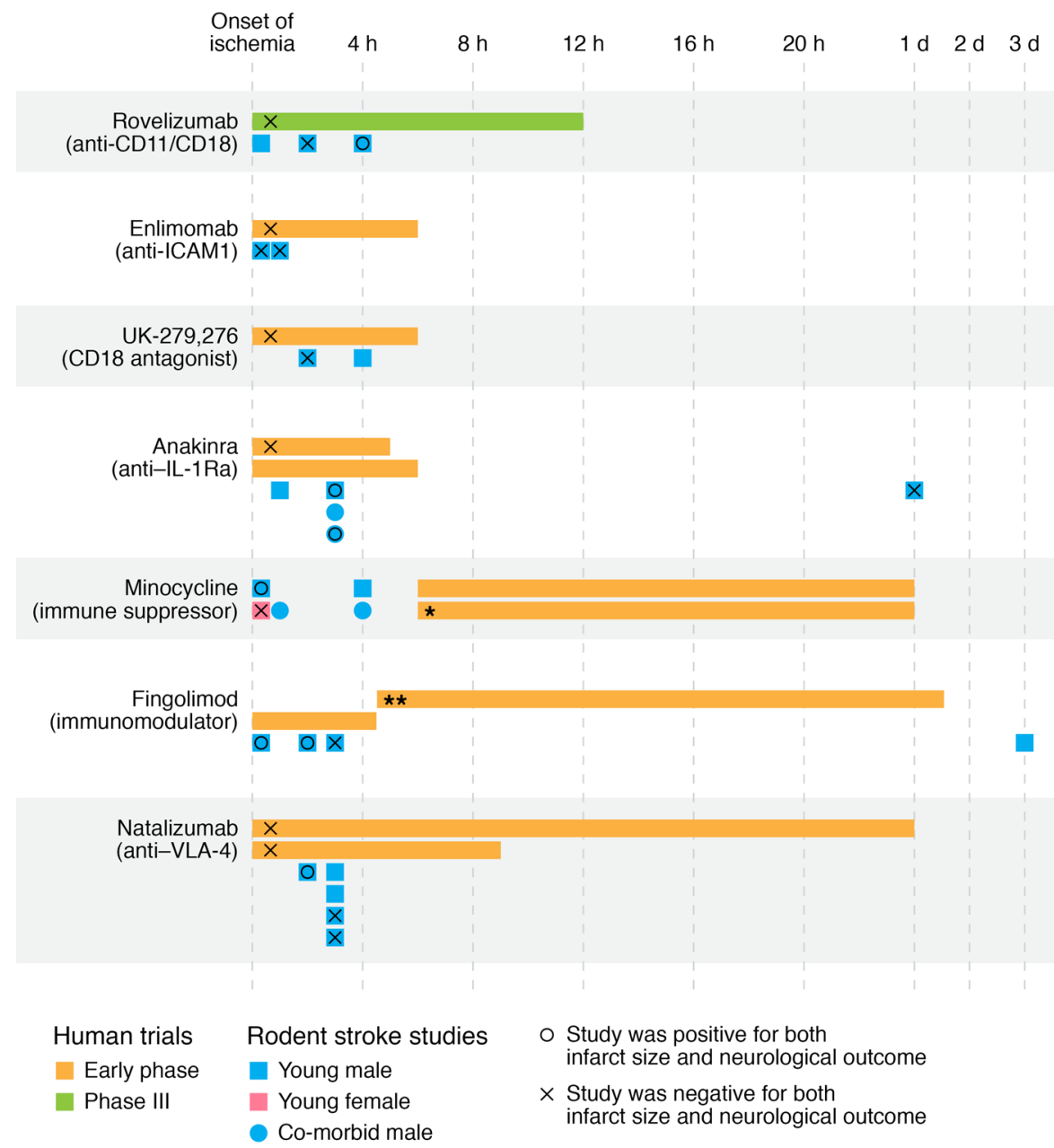

Figure 3. Timing and success of selected immunomodulatory therapies for stroke. Human studies were selected as those that were later stage and utilized immunomodulatory drugs or antibodies. For each agent, the human studies are listed in chronological order, with the length of the bars indicating the treatment period from the time the participant was last seen normal. Below the human trials are animal studies with that agent where the first dose was delivered after stroke, and either infarct size or neurological outcome was tested. If both were positive, the animal studies are marked with a circle, and if both were negative, they are marked with an $x$. In cases where only neurological outcome or only stroke size was tested, or where one was positive and the other negative, the study is not marked with a symbol. Comorbidities in animal studies were aging, diabetes, hypertension, and hypercholesterolemia. Additional details and references are in Supplemental Table 1. *This study had a positive effect in males but not females. ${ }^{*}$ Drug dose timing listed only as mean \pm SD, which is graphed here.
Stroke changes cognitive trajectory and doubles the risk of a new diagnosis of dementia (7). Both stroke risk factors and recurrent stroke may contribute to the increased dementia risk (165, 166). It is of interest that, in animal models, inflammation can cause cognitive decline with or without stroke. High-salt diet without stroke can increase peripheral Th17 cells that drive cognitive decline through a deficit in endothelial NO and accumulation of insoluble tau $(167,168)$. TLR4 signaling promotes aberrant neurogenesis that causes cognitive decline after stroke $(169,170)$. B lymphocytes, perhaps as part of an autoimmune response triggered by a proinflammatory state, also cause loss of hippocampal long-term potentiation and cognitive decline (140). In humans, an association between anti-MBP antibodies and cognitive decline has been shown (171), and a proinflammatory state in the blood 2 days after stroke is associated with subsequent cognitive decline (152).

\section{Poststroke fatigue and depression}

Fatigue and depression often are difficult to disentangle from each other and from poststroke cognitive impairment, and are a substantial barrier to recovery and rehabilitation $(172,173)$. But, as with poststroke dementia, there are only small or single studies linking depression and fatigue to neuroinflammation after stroke (174-176), and this is a highly relevant and fertile area for future research.

\section{Postischemic immunity as a therapeutic target in humans}

Immune therapy for stroke has focused primarily on reducing injury volume and improving functional outcomes. Several drugs targeting the immune system that were effective in preclinical studies have failed in clinical trials (Figure 3 and Supplemental Table 1; supplemental material available online with this article; https://doi.org/10.1172/JCI135530DS1), and there is no currently FDA-approved immunomodulatory drug for stroke. There are two FDA-approved immunomodulatory drugs with extensive safety records that may be beneficial in stroke: Fingolimod, a sphingosine-1 phosphate receptor modulator that prevents lymphocyte egress from lymph nodes, was approved by the FDA for multiple sclerosis (177). A small study demonstrated better 90-day outcomes by Barthel index and modified Rankin scale in 22 patients (178), and there is an ongoing study of fingolimod in hemorrhagic stroke (179). There are also promising data for an IL-1 receptor antagonist, which reduced stroke size in one study (180) and the peripheral immune response but not stroke size in another (181).

The reasons for failure of preclinically effective agents in clinical trials in stroke have been extensively debated (182-184). One reason could be that clinical trials and animal studies have traditionally used different designs. For example, clinical trials often have a longer interval between ischemia and drug dosing than pre- 
clinical studies (Figure 3 and Supplemental Table 1) and are sometimes underpowered. On the other hand, animal studies have not always assessed efficacy in females, which constitute $50 \%$ of stroke patients, and power calculations, randomization, and blinding were not always implemented (184). Preclinical studies are generally performed in young adults without comorbidities, whereas most stroke patients are older, with high rates of obesity, hypertension, hyperlipidemia, and diabetes. Conventional stroke risk factors and infections have profound impact on the immune system and stroke outcome (185), and need to be incorporated into preclinical models (186). Reproducibility and publication bias against negative results have also emerged as a problem in preclinical research $(184,187)$. However, there have been increasing calls to harmonize the design of clinical and preclinical animal trials, include both sexes, use aged animals with comorbidities, and implement steps to increase reproducibility $(188,189)$. These approaches have started to pay off. For example, an IL-1 receptor antagonist demonstrated benefit in aged obese rats with dyslipidemia $(190,191)$. Furthermore, a randomized multicentric preclinical trial using antibodies against VLA-4 reduced brain injury only in small infarcts caused by permanent ischemia (192). This result suggests that immunomodulators may be effective only in certain types of strokes, which may help explain the failure of the clinical trial using VLA-4 antibodies (193).

\section{Conclusions and future directions}

The data reviewed above provide strong evidence of an important role of the immune system in acute and chronic phases of ischemic damage, as well as in the long-term sequelae of stroke. Cerebral ischemic injury engages both innate and adaptive immunity, in the brain and systemically. The ensuing immune response plays a key role in stroke outcome. In the past, several clinical trials attempted to target postischemic immune responses with disappointing results, in part due to an incomplete understanding of poststroke immunity (19). Fortunately, our understanding of the immunology of stroke has improved, and we have gained a more nuanced appreciation for the diverse roles that innate and adaptive immunity plays in stroke. For example, the powerful innate immune response to acute ischemia is damaging to the brain in the acute phase of stroke, but in the late phase, Tregs, microglia, and macrophages may be protective and set the stage for postischemic repair processes. Accordingly, future clinical trials must take into consideration the double-edged role of the immune system, and treatments must be tailored to the specific stage of tissue damage evolution and inflammatory response and would benefit from imaging approaches assessing inflammation (194). These clinical efforts would benefit from animal studies examining in greater detail the signaling mechanisms that shift immune responses from deleterious to beneficial. Gaining better insight into the interaction between systemic immunity and the brain, in animal models as in human stroke, is an important area of research. Systemic lymphoid organs and the gut are influenced by stroke, with profound effects on brain damage evolution. Changes in systemic immunity may also impact poststroke infections, and the role of gut bacteria as an infectious source should be investigated.

Stroke therapy changed dramatically in 2018 with the DAWN and DEFUSE 3 intravascular thrombectomy trials (Table 1 and refs. 195, 196). Sophisticated real-time imaging techniques can now detect salvageable tissue that is not functional but not yet dead, marked by low but not lethally reduced blood flow (penumbra). Whether and how post-thrombectomy reperfusion reshapes immune responses after stroke remain to be established. After reperfusion, circulating white cells may gain better access to the brain, which may modify tissue outcome. In about $50 \%$ of patients, arterial recanalization does not restore tissue perfusion and neurological function (futile recanalization) (197); the role of innate immunity in this process remains unexplored. Furthermore, it will be interesting to assess the impact of mechanical thrombectomy on adaptive immunity and poststroke sequelae. Fatigue, depression, and cognitive decline are large problems for stroke survivors that may be linked to inflammation. This issue is particularly pressing in light of the anticipated increase in stroke prevalence due to reduced mortality in the acute phase (see Introduction). Despite recovery of sensorimotor and language deficits, neuropsychiatric sequelae have a paramount negative impact on the quality of life of stroke survivors. Animal and human studies addressing the role of adaptive immunity and chronic inflammation in these long-term sequelae would advance our understanding of these poorly studied phenomena and suggest new therapeutic approaches to be tested in clinical trials.

In this regard, animal studies should focus more on the neuropsychiatric consequences of stroke. Considering the complex innate and adaptive immune changes occurring in stroke, treatments targeting the immune system may be beneficial in reducing injury volume and motor deficits, but may not affect, and may even exacerbate, long-term sequelae. In addition, these long-term effects are more likely to occur with comorbidities and recurrent strokes, and should be routinely evaluated in animal studies. Addressing these critical issues will require concerted effort by both basic and clinical neuroscientists to harmonize protocols and approaches between experimental studies and clinical trials. On the one hand, basic science studies should attempt to better mimic the complexity of human trials by using models incorporating aging and comorbidities, studying both sexes and long-term sequelae, and adopting randomization, blinding, and data analysis protocols comparable to those of clinical trials. On the other hand, clinical trials should be aware of the therapeutic window of the agents used, confirm salvageable tissue and target engagement, and be adequately powered to assess efficacy in stroke subtypes. Questions raised by clinical trials can then be brought back to the bench for mechanistic investigations to refine subsequent trials and maximize potential beneficial effects. Such synergistic interaction between basic and clinical studies would be particularly valuable in efforts to modulate immunity for therapeutic purposes and advance stroke therapy to the next level.

\section{Acknowledgments}

CI is supported by NIH grant R01-NS34179, JA by R01-NS081179 and R01-NS09450, and MSB by a Frontiers in Brain Health Award from the American Heart Association/Paul Allen Foundation (grant 19PABHI34580007).

Address correspondence to: Costantino Iadecola, Feil Family Brain and Mind Research Institute, 407 East 61st Street, RR-303, New York, New York 10065, USA. Phone: 646.962.8279; Email: coi2001@med.cornell.edu. 
1. GBD 2016 Stroke Collaborators. Global, regional, and national burden of stroke, 1990-2016: a systematic analysis for the Global Burden of Disease Study 2016. Lancet Neurol. 2019;18(5):439-458.

2. Mensah GA, et al. Decline in cardiovascular mortality: possible causes and implications. Circ Res. 2017;120(2):366-380

3. GBD 2016 Lifetime Risk of Stroke Collaborators, et al. Global, Regional, and Country-Specific Lifetime Risks of Stroke, 1990 and 2016. N Engl J Med. 2018;379(25):2429-2437.

4. Ekker MS, Verhoeven JI, Vaartjes I, van Nieuwenhuizen KM, Klijn CJM, de Leeuw FE. Stroke incidence in young adults according to age, subtype, sex, and time trends. Neurology. 2019;92(21):e2444-e2454.

5. Bhatt N, Malik AM, Chaturvedi S. Stroke in young adults: five new things. Neurol Clin Pract. 2018;8(6):501-506.

6. Ovbiagele B, et al. Forecasting the future of stroke in the United States: a policy statement from the American Heart Association and American Stroke Association. Stroke. 2013;44(8):2361-2375.

7. Kuźma E, Lourida I, Moore SF, Levine DA, Ukoumunne OC, Llewellyn DJ. Stroke and dementia risk: a systematic review and meta-analysis. Alzheimers Dement. 2018;14(11):1416-1426.

8. Toledo JB, et al. Contribution of cerebrovascular disease in autopsy confirmed neurodegenerative disease cases in the National Alzheimer's Coordinating Centre. Brain. 2013;136(pt 9):2697-2706

9. Hachinski V, et al. Preventing dementia by preventing stroke: the Berlin Manifesto. Alzheimers Dement. 2019;15(7):961-984.

10. Campbell BCV, et al. Ischaemic stroke. Nat Rev Dis Primers. 2019;5(1):70.

11. Iadecola $\mathrm{C}$, et al. Vascular cognitive impairment and dementia: JACC Scientific Expert Panel. JAm Coll Cardiol. 2019;73(25):3326-3344.

12. Gross BA, Jankowitz BT, Friedlander RM. Cerebral intraparenchymal hemorrhage: a review. JAMA. 2019;321(13):1295-1303.

13. Lawton MT, Vates GE. Subarachnoid hemorrhage. N Engl JMed. 2017;377(3):257-266

14. Katan M, Luft A. Global burden of stroke. Semin Neurol. 2018;38(2):208-211.

15. Ribeiro $\mathrm{M}$, et al. Meningeal $\gamma \delta \mathrm{T}$ cell-derived IL-17 controls synaptic plasticity and short-term memory. Sci Immunol. 2019;4(40):eaay5199.

16. Kipnis J. Multifaceted interactions between adaptive immunity and the central nervous system. Science. 2016;353(6301):766-771.

17. Engelhardt B, Vajkoczy P, Weller RO. The movers and shapers in immune privilege of the CNS. Nat Immunol. 2017;18(2):123-131.

18. Hammond TR, Marsh SE, Stevens B. Immune signaling in neurodegeneration. Immunity. 2019;50(4):955-974.

19. Iadecola C, Anrather J. The immunology of stroke: from mechanisms to translation. Nat Med. 2011;17(7):796-808.

20. Bruetman ME, Fields WS, Crawford ES, Debakey ME. Cerebral hemorrhage in carotid artery surgery. Arch Neurol. 1963;9:458-467.

21. Astrup J, Siesjö B, Symon L. Thresholds in cerebral ischemia-the ischemic penumbra. Stroke. 1981;6:723-725.

22. Baron JC. Protecting the ischaemic penumbra as an adjunct to thrombectomy for acute stroke. $\mathrm{Nat}$ Rev Neurol. 2018;14(6):325-337.

23. Moskowitz MA, Lo EH, Iadecola C. The science of stroke: mechanisms in search of treatments. Neuron. 2010;67(2):181-198.

24. Gauberti M, De Lizarrondo SM, Vivien D. The "inflammatory penumbra" in ischemic stroke: from clinical data to experimental evidence. Eur Stroke J. 2016;1(1):20-27.

25. Weinstein PR, Hong S, Sharp FR. Molecular identification of the ischemic penumbra. Stroke. 2004;35(11 suppl 1):2666-2670.

26. Dirnagl U, Iadecola C, Moskowitz MA. Pathobiology of ischaemic stroke: an integrated view. Trends Neurosci. 1999;22(9):391-397.

27. National Institute of Neurological Disorders Stroke rt-PA Stroke Study Group. Tissue plasminogen activator for acute ischemic stroke. $N$ Engl $J$ Med.1995;333(24):1581-1587.

28. Dmytriw AA, Zhang Y, Mendes Pereira V. Mechanical thrombectomy and the future of acute stroke treatment. Eur J Radiol. 2019;112:214-221.

29. Mendez AA, et al. Update in the early management and reperfusion strategies of patients with acute ischemic stroke. Crit Care Res Pract. 2018;2018:9168731.

30. Rinaldo L, Rabinstein AA, Cloft H, Knudsen JM, Castilla LR, Brinjikji W. Racial and ethnic disparities in the utilization of thrombectomy for acute stroke. Stroke. 2019;50(9):2428-2432.

31. Hill MD, et al. Efficacy and safety of nerinetide for the treatment of acute ischaemic stroke (ESCAPE-NA1): a multicentre, doubleblind, randomised controlled trial. Lancet. 2020;395(10227):878-887.

32. Jain A, Pasare C. Innate control of adaptive immunity: beyond the three-signal paradigm. JImmunol. 2017;198(10):3791-3800.

33. Abbas AK, Lichtman AH, Pillai S. Cellular and Molecular Immunology. 7th ed. Philadelphia, Pennsylvania, USA: Elsevier; 2012.

34. Yilmaz G, Granger DN. Leukocyte recruitment and ischemic brain injury. Neuromolecular Med 2010;12(2):193-204.

35. del Zoppo GJ, Schmid-Schönbein GW, Mori E, Copeland BR, Chang CM. Polymorphonuclear leukocytes occlude capillaries following middle cerebral artery occlusion and reperfusion in baboons. Stroke. 1991;22(10):1276-1283.

36. De Meyer SF, Denorme F, Langhauser F, Geuss E, Fluri F, Kleinschnitz C. Thromboinflammation in stroke brain damage. Stroke. 2016;47(4):1165-1172.

37. Rezaie AR. Protease-activated receptor signalling by coagulation proteases in endothelial cells. Thromb Haemost. 2014;112(5):876-882.

38. Amara U, et al. Interaction between the coagulation and complement system. Adv Exp Med Biol. 2008;632:71-79.

39. Széplaki G, et al. Strong complement activation after acute ischemic stroke is associated with unfavorable outcomes. Atherosclerosis. 2009;204(1):315-320.

40. Schäfer MK, et al. Complement C1q is dramatically up-regulated in brain microglia in response to transient global cerebral ischemia. J Immunol. 2000;164(10):5446-5452.

41. Mocco J, et al. Complement component C3 mediates inflammatory injury following focal cerebral ischemia. Circ Res. 2006;99(2):209-217.

42. Orsini F, et al. Mannose-binding lectin drives platelet inflammatory phenotype and vascular damage after cerebral ischemia in mice via IL (interleukin)-1 $\alpha$. Arterioscler Thromb Vasc Biol. 2018;38(11):2678-2690.

43. Cervera A, et al. Genetically-defined deficiency of mannose-binding lectin is associated with protection after experimental stroke in mice and outcome in human stroke. PLoS One. 2010;5(2):e8433

44. Osthoff $\mathrm{M}$, et al. Mannose-binding lectin deficiency is associated with smaller infarction size and favorable outcome in ischemic stroke patients. PLoS One. 2011;6(6):e21338.

45. Alawieh A, Langley EF, Tomlinson S. Targeted complement inhibition salvages stressed neurons and inhibits neuroinflammation after stroke in mice. Sci Transl Med. 2018;10(441):eaao6459.

46. Rupalla K, Allegrini PR, Sauer D, Wiessner C. Time course of microglia activation and apoptosis in various brain regions after permanent focal cerebral ischemia in mice. Acta Neuropathol. 1998;96(2):172-178.

47. Morrison HW, Filosa JA. A quantitative spatiotemporal analysis of microglia morphology during ischemic stroke and reperfusion. J Neuroinflammation. 2013;10:4.

48. Schilling M, Besselmann M, Leonhard C, Mueller M, Ringelstein EB, Kiefer R. Microglial activation precedes and predominates over macrophage infiltration in transient focal cerebral ischemia: a study in green fluorescent protein transgenic bone marrow chimeric mice. Exp Neurol. 2003;183(1):25-33.

49. Lalancette-Hébert M, Gowing G, Simard A, Weng YC, Kriz J. Selective ablation of proliferating microglial cells exacerbates ischemic injury in the brain. J Neurosci. 2007;27(10):2596-2605.

50. Jin WN, et al. Depletion of microglia exacerbates postischemic inflammation and brain injury. JCereb Blood Flow Metab. 2017;37(6):2224-2236.

51. Kawabori M, et al. Triggering receptor expressed on myeloid cells 2 (TREM2) deficiency attenuates phagocytic activities of microglia and exacerbates ischemic damage in experimental stroke. J Neurosci. 2015;35(8):3384-3396.

52. Otxoa-de-Amezaga A, et al. Microglial cell loss after ischemic stroke favors brain neutrophil accumulation. Acta Neuropathol. 2019;137(2):321-341.

53. Gelderblom M, et al. Neutralization of the IL-17 axis diminishes neutrophil invasion and protects from ischemic stroke. Blood. 2012;120(18):3793-3802.

54. Zamanian JL, et al. Genomic analysis of reactive astrogliosis. J Neurosci. 2012;32(18):6391-6410.

55. Pedragosa J, et al. CNS-border associated macrophages respond to acute ischemic stroke attracting granulocytes and promoting vascular leakage. Acta Neuropathol Commun. 2018;6(1):76.

56. Lindsberg PJ, Strbian D, Karjalainen-Lindsberg ML. Mast cells as early responders in the regulation of acute blood-brain barrier changes after cerebral ischemia and hemorrhage. J Cereb Blood Flow Metab. 2010;30(4):689-702.

57. Strbian D, Karjalainen-Lindsberg ML, Tatlisumak T, Lindsberg PJ. Cerebral mast cells 
regulate early ischemic brain swelling and neutrophil accumulation. JCereb Blood Flow Metab. 2006;26(5):605-612.

58. Greter M, et al. Stroma-derived interleukin-34 controls the development and maintenance of langerhans cells and the maintenance of microglia. Immunity. 2012;37(6):1050-1060.

59. Wang Y, et al. IL-34 is a tissue-restricted ligand of CSF1R required for the development of Langerhans cells and microglia. Nat Immunol. 2012;13(8):753-760.

60. van der Maten G, Henck V, Wieloch T, Ruscher $\mathrm{K}$. $\mathrm{CX}_{3} \mathrm{C}$ chemokine receptor 1 deficiency modulates microglia morphology but does not affect lesion size and short-term deficits after experimental stroke. BMC Neurosci. 2017;18(1):11.

61. Yang Y, et al. Loss of neuronal CD200 contributed to microglial activation after acute cerebral ischemia in mice. Neurosci Lett. 2018;678:48-54.

62. Tang Z, et al. CX3CR1 deficiency suppresses activation and neurotoxicity of microglia/macrophage in experimental ischemic stroke. J Neuroinflammation. 2014;11:26.

63. Cisbani G, Le Behot A, Plante MM, Préfontaine $\mathrm{P}$, Lecordier M, Rivest S. Role of the chemokine receptors CCR2 and CX3CR1 in an experimental model of thrombotic stroke. Brain Behav Immun. 2018;70:280-292.

64. Wang J, et al. Ischemia-induced neuronal cell death is mediated by chemokine receptor CX3CR1. Sci Rep. 2018;8(1):556.

65. Allen C, et al. Neutrophil cerebrovascular transmigration triggers rapid neurotoxicity through release of proteases associated with decondensed DNA. JImmunol. 2012;189(1):381-392.

66. Garcia-Bonilla L, et al. Inducible nitric oxide synthase in neutrophils and endothelium contributes to ischemic brain injury in mice. JImmunol. 2014;193(5):2531-2537.

67. Kim SW, Lee H, Lee HK, Kim ID, Lee JK. Neutrophil extracellular trap induced by HMGB1 exacerbates damages in the ischemic brain. Acta Neuropathol Commun. 2019;7(1):94.

68. Stowe AM, et al. Neutrophil elastase and neurovascular injury following focal stroke and reperfusion. Neurobiol Dis. 2009;35(1):82-90.

69. Cuartero MI, et al. N2 neutrophils, novel players in brain inflammation after stroke: modulation by the PPAR $\gamma$ agonist rosiglitazone. Stroke. 2013;44(12):3498-3508.

70. García-Culebras A, et al. Role of TLR4 (Toll-like receptor 4) in N1/N2 neutrophil programming after stroke. Stroke. 2019;50(10):2922-2932.

71. Garcia-Bonilla L, et al. Spatio-temporal profile, phenotypic diversity, and fate of recruited monocytes into the post-ischemic brain. J Neuroinflammation. 2016;13(1):285.

72. Gliem M, et al. Macrophages prevent hemorrhagic infarct transformation in murine stroke models. Ann Neurol. 2012;71(6):743-752.

73. Wattananit S, et al. Monocyte-derived macrophages contribute to spontaneous long-term functional recovery after stroke in mice. JNeurosci. 2016;36(15):4182-4195.

74. Hu X, et al. Microglia/macrophage polarization dynamics reveal novel mechanism of injury expansion after focal cerebral ischemia. Stroke. 2012;43(11):3063-3070.
75. Miró-Mur F, et al. Immature monocytes recruited to the ischemic mouse brain differentiate into macrophages with features of alternative activation. Brain Behav Immun. 2016;53:18-33.

76. Perego C, Fumagalli S, De Simoni MG. Temporal pattern of expression and colocalization of microglia/macrophage phenotype markers following brain ischemic injury in mice. JNeuroinflammation. 2011;8:174.

77. Pedragosa J, et al. CCR2 deficiency in monocytes impairs angiogenesis and functional recovery after ischemic stroke in mice [published online ahead of print March 9, 2020]. JCereb Blood Flow Metab. https://doi.org/10.1177/027167 8X20909055.

78. Bao Y, Kim E, Bhosle S, Mehta H, Cho S. A role for spleen monocytes in post-ischemic brain inflammation and injury. J Neuroinflammation. 2010;7:92.

79. Kim E, Yang J, Beltran CD, Cho S. Role of spleen-derived monocytes/macrophages in acute ischemic brain injury. JCereb Blood Flow Metab . 2014;34(8):1411-1419.

80. Yang B, et al. Multipotent adult progenitor cells enhance recovery after stroke by modulating the immune response from the spleen. Stem Cells. 2017;35(5):1290-1302.

81. Garcia-Bonilla L, et al. Endogenous protection from ischemic brain injury by preconditioned monocytes. JNeurosci. 2018;38(30):6722-6736.

82. Chauhan A, Al Mamun A, Spiegel G, Harris N, Zhu L, McCullough LD. Splenectomy protects aged mice from injury after experimental stroke. Neurobiol Aging. 2018;61:102-111.

83. Guilliams M, et al. Dendritic cells, monocytes and macrophages: a unified nomenclature based on ontogeny. Nat Rev Immunol. 2014;14(8):571-578.

84. Felger JC, et al. Brain dendritic cells in ischemic stroke: time course, activation state, and origin. Brain Behav Immun. 2010;24(5):724-737.

85. Gelderblom M, et al. IL-23 (interleukin-23)-producing conventional dendritic cells control the detrimental IL-17 (interleukin-17) response in stroke. Stroke. 2018;49(1):155-164.

86. Yilmaz A, et al. Transient decrease in circulating dendritic cell precursors after acute stroke: potential recruitment into the brain. Clin Sci. 2009;118(2):147-157.

87. Hurn PD, et al. T- and B-cell-deficient mice with experimental stroke have reduced lesion size and inflammation. JCereb Blood Flow Metab. 2007;27(11):1798-1805

88. Kleinschnitz C, et al. Early detrimental T-cell effects in experimental cerebral ischemia are neither related to adaptive immunity nor thrombus formation. Blood. 2010;115(18):3835-3842.

89. Benakis $\mathrm{C}$, et al. Commensal microbiota affects ischemic stroke outcome by regulating intestinal $\gamma \delta$ T cells. Nat Med. 2016;22(5):516-523.

90. Shichita T, et al. Pivotal role of cerebral interleukin-17-producing gammadeltaT cells in the delayed phase of ischemic brain injury. Nat Med. 2009;15(8):946-950.

91. Gan Y, et al. Ischemic neurons recruit natural killer cells that accelerate brain infarction. Proc Natl Acad Sci U S A. 2014;111(7):2704-2709.

92. Liesz A, et al. Regulatory T cells are key cerebroprotective immunomodulators in acute experi- mental stroke. Nat Med. 2009;15(2):192-199.

93. Knowland D, et al. Stepwise recruitment of transcellular and paracellular pathways underlies blood-brain barrier breakdown in stroke. Neuron. 2014;82(3):603-617.

94. Yang Y, Rosenberg GA. Blood-brain barrier breakdown in acute and chronic cerebrovascular disease. Stroke. 2011;42(11):3323-3328.

95. Garcia-Bonilla L, Racchumi G, Murphy M, Anrather J, Iadecola C. Endothelial CD36 contributes to postischemic brain injury by promoting neutrophil activation via CSF3. J Neurosci. 2015;35(44):14783-14793.

96. Otxoa-de-Amezaga A, et al. Location of neutrophils in different compartments of the damaged mouse brain after severe ischemia/reperfusion. Stroke. 2019;50(6):1548-1557.

97. Perez-de-Puig I, et al. Neutrophil recruitment to the brain in mouse and human ischemic stroke. Acta Neuropathol. 2015;129(2):239-257.

98. Enzmann G, et al. The neurovascular unit as a selective barrier to polymorphonuclear granulocyte (PMN) infiltration into the brain after ischemic injury. Acta Neuropathol. 2013;125(3):395-412.

99. Garcia JH, Liu KF, Yoshida Y, Lian J, Chen S, del Zoppo GJ. Influx of leukocytes and platelets in an evolving brain infarct (Wistar rat). Am J Pathol. 1994;144(1):188-199.

100.Cai R, et al. Panoptic imaging of transparent mice reveals whole-body neuronal projections and skull-meninges connections. Nat Neurosci. 2019;22(2):317-327.

101. Herisson F, et al. Direct vascular channels connect skull bone marrow and the brain surface enabling myeloid cell migration. Nat Neurosci. 2018;21(9):1209-1217.

102. Kivisäkk P, et al. Human cerebrospinal fluid central memory CD4+ T cells: evidence for trafficking through choroid plexus and meninges via P-selectin. Proc Natl Acad Sci U S A. 2003;100(14):8389-8394.

103.Steffen BJ, Breier G, Butcher EC, Schulz M, Engelhardt B. ICAM-1, VCAM-1, and MAdCAM- 1 are expressed on choroid plexus epithelium but not endothelium and mediate binding of lymphocytes in vitro. Am J Pathol. 1996;148(6):1819-1838.

104. Reboldi A, et al. C-C chemokine receptor 6-regulated entry of TH-17 cells into the CNS through the choroid plexus is required for the initiation of EAE. Nat Immunol. 2009;10(5):514-523.

105. Ge R, et al. Choroid plexus-cerebrospinal fluid route for monocyte-derived macrophages after stroke. J Neuroinflammation. 2017;14(1):153.

106.Shechter R, et al. Recruitment of beneficial M2 macrophages to injured spinal cord is orchestrated by remote brain choroid plexus. Immunity . 2013;38(3):555-569.

107. Petrovic-Djergovic D, et al. Tissue-resident ecto-5' nucleotidase (CD73) regulates leukocyte trafficking in the ischemic brain. JImmunol. 2012;188(5):2387-2398.

108. Chinnery HR, Ruitenberg MJ, McMenamin PG. Novel characterization of monocyte-derived cell populations in the meninges and choroid plexus and their rates of replenishment in bone marrow chimeric mice. J Neuropathol Exp Neurol. 2010;69(9):896-909.

109. Bower NI, Hogan BM. Brain drains: new insights 
into brain clearance pathways from lymphatic biology. J Mol Med. 2018;96(5):383-390.

110. Huang JM, Hu J, Chen N, Hu ML. Relationship between plasma high-mobility group box-1 levels and clinical outcomes of ischemic stroke. JCrit Care. 2013;28(5):792-797.

111. Liesz A, et al. DAMP signaling is a key pathway inducing immune modulation after brain injury. J Neurosci. 2015;35(2):583-598.

112. Esposito E, et al. Brain-to-cervical lymph node signaling after stroke. Nat Commun. 2019;10(1):5306.

113. Chapman KZ, et al. A rapid and transient peripheral inflammatory response precedes brain inflammation after experimental stroke. J Cereb Blood Flow Metab. 2009;29(11):1764-1768.

114. Offner H, Subramanian S, Parker SM, Afentoulis ME, Vandenbark AA, Hurn PD. Experimental stroke induces massive, rapid activation of the peripheral immune system. J Cereb Blood Flow Metab. 2006;26(5):654-665.

115. Ferrarese $\mathrm{C}$, et al. Increased cytokine release from peripheral blood cells after acute stroke. JCereb Blood Flow Metab. 1999;19(9):1004-1009.

116. Basic Kes V, Simundic AM, Nikolac N, Topic E, Demarin V. Pro-inflammatory and anti-inflammatory cytokines in acute ischemic stroke and their relation to early neurological deficit and stroke outcome. Clin Biochem. 2008;41(16-17):1330-1334.

117. Du X, et al. Genomic profiles for human peripheral blood $\mathrm{T}$ cells, B cells, natural killer cells, monocytes, and polymorphonuclear cells: comparisons to ischemic stroke, migraine, and Tourette syndrome. Genomics. 2006;87(6):693-703.

118. Tang Y, et al. Gene expression in blood changes rapidly in neutrophils and monocytes after ischemic stroke in humans: a microarray study. JCereb Blood Flow Metab. 2006;26(8):1089-1102.

119. Schulte-Herbrüggen $O$, Quarcoo $D$, Meisel A, Meisel C. Differential affection of intestinal immune cell populations after cerebral ischemia in mice. Neuroimmunomodulation. 2009;16(3):213-218.

120. Langhorne $\mathrm{P}$, et al. Medical complications after stroke: a multicenter study. Stroke. 2000;31(6):1223-1229.

121. Członkowska A, Cyrta B, Korlak J. Immunological observations on patients with acute cerebral vascular disease. J Neurol Sci. 1979;43(3):455-464.

122.Członkowska A, Korlak J, Kuczyńska A. Lymphocyte subsets after stroke. Ann N Y Acad Sci. 1988;540:608-610.

123. Prass K, et al. Stroke-induced immunodeficiency promotes spontaneous bacterial infections and is mediated by sympathetic activation reversal by poststroke Thelper cell type 1-like immunostimulation. J Exp Med. 2003;198(5):725-736.

124. Rosas-Ballina M, Tracey KJ. The neurology of the immune system: neural reflexes regulate immunity. Neuron. 2009;64(1):28-32.

125. Wong CH, Jenne CN, Lee WY, Léger C, Kubes P. Functional innervation of hepatic iNKT cells is immunosuppressive following stroke. Science. 2011;334(6052):101-105.

126. Courties G, et al. Ischemic stroke activates hematopoietic bone marrow stem cells. Circ Res. 2015;116(3):407-417.
127. Ay I, Sorensen AG, Ay H. Vagus nerve stimulation reduces infarct size in rat focal cerebral ischemia: an unlikely role for cerebral blood flow. Brain Res. 2011;1392:110-115.

128. Han Z, et al. Activation of $\alpha-7$ nicotinic acetylcholine receptor reduces ischemic stroke injury through reduction of pro-inflammatory macrophages and oxidative stress. PLoS One. 2014;9(8):e105711.

129. Hiraki T, Baker W, Greenberg JH. Effect of vagus nerve stimulation during transient focal cerebral ischemia on chronic outcome in rats. J Neurosci Res. 2012;90(4):887-894.

130. Yang Y, et al. Non-invasive vagus nerve stimulation reduces blood-brain barrier disruption in a rat model of ischemic stroke. Brain Stimul. 2018;11(4):689-698.

131. Engel O, et al. Cholinergic pathway suppresses pulmonary innate immunity facilitating pneumonia after stroke. Stroke. 2015;46(11):3232-3240.

132. Singh V, et al. Microbiota dysbiosis controls the neuroinflammatory response after stroke. J Neurosci. 2016;36(28):7428-7440.

133. Camara-Lemarroy CR, Ibarra-Yruegas BE, Gongora-Rivera F. Gastrointestinal complications after ischemic stroke. J Neurol Sci. 2014;346(1-2):20-25.

134. Houlden A, et al. Brain injury induces specific changes in the caecal microbiota of mice via altered autonomic activity and mucoprotein production. Brain Behav Immun. 2016;57:10-20.

135. Stanley D, et al. Translocation and dissemination of commensal bacteria in post-stroke infection. Nat Med. 2016;22(11):1277-1284

136. Crapser J, et al. Ischemic stroke induces gut permeability and enhances bacterial translocation leading to sepsis in aged mice. Aging (Albany NY). 2016;8(5):1049-1063.

137. Liu Q, et al. Peripheral TREM1 responses to brain and intestinal immunogens amplify stroke severity. Nat Immunol. 2019;20(8):1023-1034.

138. Planas AM, Gómez-Choco M, Urra X, Gorina R, Caballero M, Chamorro Á. Brain-derived antigens in lymphoid tissue of patients with acute stroke. J Immunol. 2012;188(5):2156-2163.

139. Ito $\mathrm{M}$, et al. Brain regulatory $\mathrm{T}$ cells suppress astrogliosis and potentiate neurological recovery. Nature. 2019;565(7738):246-250.

140. Doyle KP, et al. B-lymphocyte-mediated delayed cognitive impairment following stroke. J Neurosci. 2015;35(5):2133-2145.

141. Ren X, et al. Regulatory B cells limit CNS inflammation and neurologic deficits in murine experimental stroke. J Neurosci. 2011;31(23):8556-8563.

142.Bodhankar S, Chen Y, Vandenbark AA, Murphy SJ, Offner H. IL-10-producing B-cells limit CNS inflammation and infarct volume in experimental stroke. Metab Brain Dis. 2013;28(3):375-386.

143. Javidi E, Magnus T. Autoimmunity after ischemic stroke and brain injury. Front Immunol. 2019;10:686.

144. Ortega SB, et al. Stroke induces a rapid adaptive autoimmune response to novel neuronal antigens. Discov Med. 2015;19(106):381-392.

145. Youngchaiyud U, Coates AS, Whittingham S, Mackay IR. Cellular-immune response to myelin protein: absence in multiple sclerosis and presence in cerebrovascular accidents. Aust NZJ
Med.1974;4(6):535-538.

146. Frenkel D, Huang Z, Maron R, Koldzic DN, Moskowitz MA, Weiner HL. Neuroprotection by IL-10-producing MOG CD4+ T cells following ischemic stroke. J Neurol Sci. 2005;233(1-2):125-132.

147. Becker KJ, Kindrick DL, Lester MP, Shea C, Ye ZC. Sensitization to brain antigens after stroke is augmented by lipopolysaccharide. JCereb Blood Flow Metab. 2005;25(12):1634-1644.

148. Becker KJ, et al. Autoimmune responses to the brain after stroke are associated with worse outcome. Stroke. 2011;42(10):2763-2769.

149. Prüss H, et al. Evidence of intrathecal immunoglobulin synthesis in stroke: a cohort study. Arch Neurol. 2012;69(6):714-717.

150. Tsementzis SA, Chao SW, Hitchcock ER, Gill JS, Beevers DG. Oligoclonal immunoglobulin $\mathrm{G}$ in acute subarachnoid hemorrhage and stroke. Neurology. 1986;36(3):395-397.

151. Süße M, Hannich MJ, Holbe C, Ruhnau J, von Sarnowski B, Dressel A. Intrathecal inflammation in young stroke. Acta Neurol Scand. 2019;140(1):9-16.

152. Tsai AS, et al. A year-long immune profile of the systemic response in acute stroke survivors. Brain. 2019;142(4):978-991.

153. Oto J, et al. Plasma proinflammatory and antiinflammatory cytokine and catecholamine concentrations as predictors of neurological outcome in acute stroke patients. JAnesth. 2008;22(3):207-212.

154. Mena H, Cadavid D, Rushing EJ. Human cerebral infarct: a proposed histopathologic classification based on 137 cases. Acta Neuropathol. 2004;108(6):524-530.

155. Chung AG, et al. Liquefaction of the brain following stroke shares a similar molecular and morphological profile with atherosclerosis and mediates secondary neurodegeneration in an osteopontin-dependent mechanism. eNeuro. 2018;5(5):ENEURO.0076-18.2018.

156.Zbesko JC, et al. Glial scars are permeable to the neurotoxic environment of chronic stroke infarcts. Neurobiol Dis. 2018;112:63-78.

157. Veldsman M. Brain atrophy estimated from structural magnetic resonance imaging as a marker of large-scale network-based neurodegeneration in aging and stroke. Geriatrics (Basel) 2017;2(4):E34.

158. Seshadri S, et al. Stroke risk profile, brain volume, and cognitive function: the Framingham Offspring Study. Neurology. 2004;63(9):1591-1599.

159. Firbank MJ, Wiseman RM, Burton EJ, Saxby BK, O'Brien JT, Ford GA. Brain atrophy and white matter hyperintensity change in older adults and relationship to blood pressure. Brain atrophy, WMH change and blood pressure. J Neurol. 2007;254(6):713-721.

160. Nagai M, Hoshide S, Ishikawa J, Shimada K, Kario K. Insular cortex atrophy as an independent determinant of disrupted diurnal rhythm of ambulatory blood pressure in elderly hypertension. Am J Hypertens. 2009;22(7):723-729.

161. Knecht S, et al. Atrial fibrillation in stroke-free patients is associated with memory impairment and hippocampal atrophy. Eur Heart J. 2008;29(17):2125-2132.

162.Lane CA, et al. Associations between blood pressure across adulthood and late-life brain structure and pathology in the neuroscience 
substudy of the 1946 British birth cohort (Insight 46): an epidemiological study. Lancet Neurol. 2019;18(10):942-952.

163. Firbank MJ, et al. Medial temporal atrophy rather than white matter hyperintensities predict cognitive decline in stroke survivors. Neurobiol Aging. 2007;28(11):1664-1669.

164. Kliper E, et al. Cognitive decline after stroke: relation to inflammatory biomarkers and hippocampal volume. Stroke. 2013;44(5):1433-1435.

165.Pendlebury ST, Rothwell PM. Prevalence, incidence, and factors associated with prestroke and post-stroke dementia: a systematic review and meta-analysis. Lancet Neurol. 2009;8(11):1006-1018.

166.Pendlebury ST, Rothwell PM, Oxford Vascular Study. Incidence and prevalence of dementia associated with transient ischaemic attack and stroke: analysis of the population-based Oxford Vascular Study. Lancet Neurol. 2019;18(3):248-258.

167. Faraco G, et al. Dietary salt promotes neurovascular and cognitive dysfunction through a gut-initiated TH17 response. Nat Neurosci. 2018;21(2):240-249.

168. Faraco $\mathrm{G}$, et al. Dietary salt promotes cognitive impairment through tau phosphorylation. Nature. 2019;574(7780):686-690.

169. Cuartero MI, et al. Abolition of aberrant neurogenesis ameliorates cognitive impairment after stroke in mice. J Clin Invest. 2019;129(4):1536-1550.

170.Caso JR, Pradillo JM, Hurtado O, Lorenzo P, Moro MA, Lizasoain I. Toll-like receptor 4 is involved in brain damage and inflammation after experimental stroke. Circulation. 2007;115(12):1599-1608.

171. Becker KJ, Tanzi P, Zierath D, Buckwalter MS. Antibodies to myelin basic protein are associated with cognitive decline after stroke. J Neuroimmunol. 2016;295-296:9-11.

172. Hinkle JL, et al. Poststroke fatigue: emerging evidence and approaches to management: a scientific statement for healthcare professionals from the American Heart Association. Stroke. 2017;48(7):e159-e170.

173. Towfighi A, et al. Poststroke depression: a scientific statement for healthcare professionals from the American Heart Association/American
Stroke Association. Stroke. 2017;48(2):e30-e43.

174. Ormstad H, Aass HC, Amthor KF, LundSørensen N, Sandvik L. Serum cytokine and glucose levels as predictors of poststroke fatigue in acute ischemic stroke patients. JNeurol. 2011;258(4):670-676.

175. Kim JM, et al. Associations of cytokine gene polymorphisms with post-stroke depression. World J Biol Psychiatry. 2012;13(8):579-587.

176. Su JA, Chou SY, Tsai CS, Hung TH. Cytokine changes in the pathophysiology of poststroke depression. Gen Hosp Psychiatry. 2012;34(1):35-39.

177. Kappos L, et al. A placebo-controlled trial of oral fingolimod in relapsing multiple sclerosis. $N$ Engl JMed. 2010;362(5):387-401.

178. Fu Y, et al. Impact of an immune modulator fingolimod on acute ischemic stroke. Proc Natl Acad Sci U S A. 2014;111(51):18315-18320.

179. Fu Y, et al. Fingolimod for the treatment of intracerebral hemorrhage: a 2-arm proof-of-concept study. JAMA Neurol. 2014;71(9):1092-1101.

180. Emsley HC, et al. A randomised phase II study of interleukin-1 receptor antagonist in acute stroke patients. J Neurol Neurosurg Psychiatry. 2005;76(10):1366-1372.

181. Smith CJ, et al. SCIL-STROKE (Subcutaneous Interleukin-1 Receptor Antagonist in Ischemic Stroke): a randomized controlled phase 2 trial. Stroke. 2018;49(5):1210-1216.

182.Pound P, Ram R. Are researchers moving away from animal models as a result of poor clinical translation in the field of stroke? An analysis of opinion papers. BMJOpen. 2020;4:e10041.

183. Dirnagl U. Thomas Willis Lecture: Is translational stroke research broken, and if so, how can we fix it? Stroke. 2016;47(8):2148-2153.

184. Neuhaus AA, et al. Importance of preclinical research in the development of neuroprotective strategies for ischemic stroke. JAMA Neurol. 2014;71(5):634-639.

185. Parikh NS, Merkler AE, Iadecola C. Inflammation, autoimmunity, infection, and stroke: epidemiology and lessons from therapeutic intervention. Stroke. 2020;51(3):711-718.

186. Cho S, Yang J. What do experimental models teach us about comorbidities in stroke? Stroke. 2018;49(2):501-507.
187. Jasny BR, et al. Fostering reproducibility in industry-academia research. Science. 2017;357(6353):759-761.

188. Bath PM, Macleod MR, Green AR. Emulating multicentre clinical stroke trials: a new paradigm for studying novel interventions in experimental models of stroke. Int J Stroke. 2009;4(6):471-479.

189. Dirnagl U, et al. A concerted appeal for international cooperation in preclinical stroke research. Stroke. 2013;44(6):1754-1760.

190.Pradillo JM, et al. Reparative effects of interleukin-1 receptor antagonist in young and aged/ co-morbid rodents after cerebral ischemia. Brain Behav Immun. 2017;61:117-126.

191. Pradillo JM, et al. Delayed administration of interleukin-1 receptor antagonist reduces ischemic brain damage and inflammation in comorbid rats. J Cereb Blood Flow Metab. 2012;32(9):1810-1819.

192.Llovera G, et al. Results of a preclinical randomized controlled multicenter trial (pRCT): antiCD49d treatment for acute brain ischemia. $S c i$ Transl Med. 2015;7(299):299ra121.

193. Elkins J, et al. Safety and efficacy of natalizumab in patients with acute ischaemic stroke (ACTION): a randomised, placebo-controlled, double-blind phase 2 trial. Lancet Neurol. 2017;16(3):217-226.

194. Gauberti M, Fournier AP, Docagne F, Vivien D, Martinez de Lizarrondo S. Molecular magnetic resonance imaging of endothelial activation in the central nervous system. Theranostics. 2018;8(5):1195-1212.

195. Nogueira RG, et al. Thrombectomy 6 to 24 hours after stroke with a mismatch between deficit and infarct. N EnglJ Med. 2018;378(1):11-21.

196. Albers GW, et al. Thrombectomy for stroke at 6 to 16 hours with selection by perfusion imaging. N Engl JMed. 2018;378(8):708-718.

197. Hussein HM, Saleem MA, Qureshi AI. Rates and predictors of futile recanalization in patients undergoing endovascular treatment in a multicenter clinical trial. Neuroradiology. 2018;60(5):557-563.

198.Stroke Unit Trialists' Collaboration. Organised inpatient (stroke unit) care for stroke. Cochrane Database Syst Rev. 2013;(9):CD000197. 